\title{
Barrett's esophagus: clinical features, obesity, and imaging
}

\author{
Eamonn M. M. Quigley, ${ }^{1}$ Brian C. Jacobson, ${ }^{2}$ Johannes Lenglinger, ${ }^{3}$ Joel H. Rubenstein, ${ }^{4}$ \\ Hashem El-Serag, ${ }^{5}$ Michele Cicala, ${ }^{6}$ Richard W. McCallum, ${ }^{7}$ Marc S. Levine, ${ }^{8}$ \\ and Richard M. Gore ${ }^{9}$ \\ ${ }^{1}$ Alimentary Pharmabiotic Centre, Department of Medicine, Clinical Sciences Building, Cork University Hospital, Cork, Ireland. \\ ${ }^{2}$ Section of Gastroenterology, Boston University Medical Center, Boston, Massachusetts. ${ }^{3}$ Department of Surgery, Medical \\ University of Vienna, Vienna, Austria. ${ }^{4}$ Veterans Affairs Center for Clinical Management Research, Ann Arbor, Michigan and \\ Division of Gastroenterology, University of Michigan Medical School, Ann Arbor, Michigan. ${ }^{5}$ Sections of Gastroenterology and \\ Health Services Research, The Houston Veterans Affairs Medical Center and Baylor College of Medicine, Houston, Texas. \\ ${ }^{6}$ Dipartimento di Malattie dell'Apparato Digerente, Campus Bio-Medico University, Rome, Italy. ${ }^{7}$ Department of Internal \\ Medicine, Paul L. Foster School of Medicine, Texas Tech University Health Sciences Center, El Paso, Texas. ${ }^{8}$ Department of \\ Radiology, Hospital of the University of Pennsylvania, Philadelphia, Pennsylvania. ${ }^{9}$ Department of Radiology, North Shore \\ University Health System, Evanston Hospital, University of Chicago, Evanston, Illinois
}

The following includes commentaries on clinical features and imaging of Barrett's esophagus (BE); the clinical factors that influence the development of BE; the influence of body fat distribution and central obesity; the role of adipocytokines and proinflammatory markers in carcinogenesis; the role of body mass index (BMI) in healing of Barrett's epithelium; the role of surgery in prevention of carcinogenesis in BE; the importance of double-contrast esophagography and cross-sectional images of the esophagus; and the value of positron emission tomography/computed tomography.

Keywords: Gastroesophageal reflux disease; Barrett's esophagus; adiponectin; lectin; esophageal adenocarcinoma; BMI; serum leptin; inflammation; diabetes; ghrelin; IGF-1; IGF binding protein; esophagography; esophagitis; central obesity; esophageal reflux; gastric banding; PET/CT; fluoro deoxyglucose

\section{Concise summaries}

- The major value of the barium study in patients with reflux symptoms is its ability to stratify them into various risk groups for Barrett's esophagus (BE) to determine the relative need for endoscopy and biopsy.

- Recent advances in multidetector computed tomography (MDCT), now allow routine visualization of the entire esophagus in a single breath-hold. Positron emission tomography/computed tomography (PET/CT) is not sufficiently sensitive and specific enough to differentiate BE from other benign disorders, but with technical improvements, may help detect malignant transformation and even dysplasia in patients with this disorder.

- It is striking that although retrospective studies tend to confirm an association with increasing length of the BE segment, this has not, in general, been borne out in prospective studies.
Excess body fat does raise one's risk of complicated gastroesophageal reflux disease (GERD), including $\mathrm{BE}$, and centralized fat is the predominant risk factor. However, whether this is true for both men and women equally remains unclear. Specific effects of central obesity, independent of body mass index (BMI), on reflux mechanisms potentially include elevated intragastric pressure and a disruption of the esophago-gastric junction (EGJ) integrity, but data are still scarce. There is substantial reason to suspect that circulating adipokines may promote BE.

- Alterations in levels of circulating factors related to obesity, including adipokines directly secreted from adipose tissue, are likely involved in the development of $\mathrm{BE}$ and neoplastic progression. Visceral fat is metabolically active, and has been strongly associated with low serum levels of potentially protective (e.g., adiponectin), or proinflammatory cytokines (e.g., interleukin-1 $\beta$, 
interleukin-6, and tumor necrosis factor- $\alpha$ ), which may play a role in the development of BE.

- However, although the prevalences of erosive esophagitis and BE are both associated with
BMI, there are no compelling data to suggest that healing rates are affected by BMI.

- Surgical treatment of obesity has positive effect on GERD and its complications, e.g., BE.

\section{Do particular clinical factors influence the development and extent of BE?}

\author{
Eamonn M. M. Quigley \\ e.quigley@ucc.ie
}

$\mathrm{BE}$ presents a formidable challenge to the clinician and endoscopist: on the one hand, its predilection to progress to adenocarcinoma of the esophagus has been widely publicized, whereas on the other, there is no doubt that, amidst a worldwide epidemic of GERD, BE has become very frequent. If, as indicated by a recent community survey from Sweden, for example, BE may be found among $2 \%$ of the general population, many of whom are asymptomatic, we have a very considerable challenge indeed, as only a very tiny minority of such individuals will ever progress to adenocarcinoma. It is self-evident that population screening for $\mathrm{BE}$ is not going to be a cost effective approach; ${ }^{1}$ what we need is a strategy that will permit the identification, at an appropriately early stage, of that subgroup who are at risk for progression. Can clinical features assist in either predicting the presence of $\mathrm{BE}$ or, more importantly, its risk for progression to cancer?

At endoscopy, BE is almost always identified in the context of chronic reflux disease and a hiatal hernia; it must be remembered that these individuals have been selected on the basis of chronic and often troublesome symptoms, a factor that has tended to bias the outcome of many studies in this area. The findings of the study by Chak and colleagues tend to confirm these clinical impressions: in comparison to GERD patients without BE, patients with $\mathrm{BE}$ (or adenocarcinoma of the esophagus or EGJ), were more likely to be male, older, obese, smokers, and drinkers of alcohol. It must be conceded that many of these associations were modest. BE subjects also tended to have more frequent and severe heartburn but no significant relationship between $\mathrm{BE}$ and duration of symptoms was evident. The strongest predictor of $\mathrm{BE}$ or related cancers (odds ratio 6.4) was, however, a family history of BE, or adenocarcinoma of the esophagus or EGJ. ${ }^{2}$ Relationships between symptomatic GERD and BE have been the subject of a recent meta-analysis, ${ }^{3}$ which dissects the interaction between GERD and BE further. Although confirming a relationship between GERD symptoms and long-segment BE, they found no association with short-segment $\mathrm{BE}$. This is more bad news; if short-segment BE is an important entity with a predilection to progression, looking for GERD symptoms will not be helpful in identifying it.

The lack of sufficient numbers did not permit Chak and colleagues to differentiate between those with $\mathrm{BE}$ alone and those who had progressed to cancer. ${ }^{2}$ To address this all important issue, Prasad and colleagues have recently performed a comprehensive systematic review of predictors for progression in $\mathrm{BE}$ and their results are not very encouraging. ${ }^{4}$ Yes - age, male gender, and length of $\mathrm{BE}$ segment were linked to progression to adenocarcinoma but the association was weak. Although obesity was identified as an independent risk factor for the development of adenocarcinoma of the esophagus, in Lagergren and his colleagues' study based on the Swedish national data base, ${ }^{5}$ there was not, as yet, in the judgment of Prasad and colleagues, compelling evidence to link obesity with BE progression. As in their examination of many other clinical and demographic factors (age, tobacco and alcohol use, acid suppression), Prasad and colleagues found the data limited, fraught with methodological issues (small sample sizes, study design, method, and extent of follow-up) and often conflicting with regard to both the direction and magnitude of the effect. Among endoscopic features, BE segment length does confer some, albeit inconsistent, relationship with progression. It is striking that although retrospective studies tend to confirm an association with increasing length of the BE segment, this has not, in general, been borne out in prospective studies.

Although we may think that we can identify clinical features that indicate whether a given individual may harbor BE, these very same features do not appear to be very helpful in helping us to make the 
much more important prediction: who is at risk for progression through dysplasia to adenocarcinoma. Recent meta-analyses, while revealing hints of what may be relevant, have also identified the important short-comings of existing data and should encourage all involved in this area to collaborate in large prospective studies of the natural history of this common condition; only then will be able to truly identify those clinical features that can help us to select patients for endoscopic surveillance with the expectation that we will truly impact on mortality from adenocarcinoma of the esophagus.

\section{What is the respective influence of BMI and body fat distribution on the development of BE?}

\section{Brian C. Jacobson \\ brian.jacobson@bmc.org}

It has been well established that an elevated BMI is associated with symptomatic GERD, erosive esophagitis, BE, and esophageal adenocarcinoma. Recently, investigators have begun to ask whether the actual distribution of body fat is more important than just having an excess amount. For example, it appears that central adiposity (also called "visceral adiposity") is important for the development of BE. ${ }^{6}$ Such central body fat could be causing more severe GERD simply through mechanical factors, such as a greater gastroesophageal pressure gradient. ${ }^{7}$ It also appears that central obesity may elicit more frequent transient lower esophageal sphincter relaxations. ${ }^{8}$ Finally, excess centralized fat is associated with a greater likelihood of a hiatal hernia. All of these factors play a role in the pathogenesis of GERD.

However, investigators have also begun to wonder about the role of adipocytokines, peptides produced by adipose tissue that have several effects on cell growth, apoptosis, and other potentially neoplastic factors. It is interesting to note that adipocytokines, such as adiponectin and leptin, are produced predominantly by visceral fat, the very fat that seems associated with BE. Men tend to have greater amounts of visceral fat than women, a fact that correlates nicely with the observation that men are twice as likely as women to develop $\mathrm{BE}$ and six or seven times as likely to progress to esophageal adenocarcinoma compared to women.

Investigators have noted an association between serum leptin and BE in men, with greater levels cor- relating with higher risk for BE. ${ }^{9}$ Interestingly, an inverse association was noted between serum leptin and $\mathrm{BE}$ in women, arguing that there may be gender differences in how body fat imparts risk for BE. It is possible that central fat plays a dominant role in the genesis of $\mathrm{BE}$ in men, but that other BMI-associated factors predominate in women. For example, peripheral fat (nonvisceral fat) is the predominant source of estrogens in postmenopausal women, and estrogens are associated with a greater risk of symptomatic GERD. ${ }^{10}$

In summary, it appears that excess body fat does raise one's risk of complicated GERD, including BE, and that centralized fat is the predominant risk factor. However, whether this is true for both men and women equally remains unclear.

\section{Can it be said that central obesity may worsen acid reflux leading to BE? If so, by what mechanisms?}

\section{Johannes Lenglinger}

johannes.lenglinger@meduniwien.ac.at

The continuous rise of the prevalence of both obesity and GERD in Western societies over the last three decades suggests a link between these conditions. The aim of this article is to provide a short overview of literature investigating the effects of central obesity on esophageal acid exposure and mechanisms potentially increasing reflux and thus leading to BE.

A significantly higher prevalence of reflux related symptoms in overweight and obese subjects was found in 7 of 9 cross-sectional surveys included in a meta-analysis of papers based on validated or structured questionnaires. ${ }^{11}$ Pooled data from these studies showed an adjusted odds ratio (OR) for reflux symptoms of 1.43 (95\% CI 1.16-1.77) in subjects with a BMI of 25-29.9 and 1.94 (95\% CI $1.47-$ 2.57 ) in individuals with a $\mathrm{BMI} \geq 30$. Jacobson et al. found an almost linear increase in the OR for frequent reflux symptoms with body mass BMI in women participating in the Nurses' Health Study. ${ }^{12}$ (Table 1).

An increase of esophageal acid exposure in overweight and obese patients has been described for subjects with and without symptoms suggestive of GERD. In most comparative studies, patients were stratified by BMI. Data on a specific role of central obesity are scarce. In a study of 206 patients undergoing 24 -hour esophageal $\mathrm{pH}$-monitoring by 
Table 1. Adjusted ORs (95\% confidence interval) based on data of 2,306 women reporting heartburn and/or acid regurgitation at least once a week and 3,904 asymptomatic women, adjusted for age, smoking status, total activity, daily caloric intake, intake of alcohol, coffee, tea, and chocolate, use of postmenopausal hormone therapy, use of antihypertensive or asthma medication, and presence or absence of diabetes mellitus ${ }^{11}$

\begin{tabular}{lccccccc}
\hline OR & 0.67 & 1.0 & 1.38 & 2.2 & 2.43 & 2.92 & 2.93 \\
$(95 \%$ CI $)$ & $(0.48-0.93)$ & & $(1.13-1.67)$ & $(1.81-2.66)$ & $(1.96-3.01)$ & $(2.35-3.62)$ & $(2.24-3.85)$ \\
BMI & $<20.0$ & $20.0-22.4$ & $22.5-24.9$ & $25.0-27.4$ & $27.5-29.9$ & $30.0-34.9$ & $\geq 35.0$ \\
\hline
\end{tabular}

El-Serag et al., waist circumference (WC) and BMI were positively correlated with parameters of acid reflux. ${ }^{13}$ As an example, for each unit increase of BMI, 2.76 more reflux episodes in the postprandial period were encountered $(P<0.001)$.

A $1 \mathrm{~cm}$ increase of WC was associated with 0.85 more reflux episodes $(P=0.002)$. In a multivariable linear regression model including both BMI (as categorical variable) and WC (as continous variable) a BMI $\geq 30$ was no longer associated with any parameter of acid exposure.

Potential mechanisms of how central obesity worsens acid reflux include a temporary or permanent disruption of the EGJ integrity and elevated intragastric pressure, resulting in an elevated gastroesophageal pressure gradient. In a high-resolution manometry study of 285 patients Pandolfino et al. found a significant correlation of BMI and WC with intragastric pressure (inspiration, BMI $(r=$ $0.57)$, WC $(r=0.62) P<0.0001$; expiration, BMI $(r=0.58)$, WC $(r=0.64), P<0.0001) .^{7}$ Furthermore a weaker, but still significant correlation of the separation of lower esophageal sphincter and crural diaphragm with BMI $(r=0.17, P<0.005)$ and WC $(r=0.21, P<0.001)$ could be demonstrated.

In a case control study of 197 patients newly diagnosed with specialized intestinal metaplasia and 418 GERD controls, a high waist to hip ratio (male $\geq 0.9$, female $\geq 0.8$ ) increased the risk for visible BE (adjusted OR 1.9, 95\% CI 1.0-3.5) and long segment BE (adjusted OR 4.1, 95\% CI 1.5-11.4) independent of BMI. ${ }^{14}$

In summary, it can be said that obesity is associated with a higher prevalence of reflux symptoms, increased esophageal acid exposure, and prevalence of BE. Specific effects of central obesity, independent of BMI, on reflux mechanisms potentially include elevated intragastric pressure and a disruption of the EGJ junction integrity, but data are still scarce. The association of anthropometric data with BE re- quires further study as well, as this has important implications for screening.

\section{What is the impact of proinflammatory adipocytokines and metabolic syndrome on the development of Barrett's mucosa?}

\author{
Joel H. Rubenstein \\ jhr@umich.edu
}

There is substantial reason to suspect that circulating adipokines may promote $\mathrm{BE}$. In brief, obesity is associated with both $\mathrm{BE}$ and esophageal adenocarcinoma. Although this is likely partly explained by a mechanical effect promoting GERD, and by dietary habits that promote GERD, obesity is also associated with a number of other cancers for which no known mechanical mechanism exists. It is increasingly recognized that adipose tissue is not an inert storage compartment, but rather metabolically active, secreting a number of substances. It is believed that those other cancers are promoted by adipokines secreted from adipose tissue, or other circulating factors. Adipokines secreted from visceral adipose appear to be particularly involved in inflammation. For instance, visceral adipocytes secrete TNF- $\alpha$, and $50-75 \%$ of circulating IL- 6 in obese subjects may be secreted from omental adipocytes. ${ }^{15,16}$ Inflammation is believed to be intrinsic to the development of BE.

The rapid rise in incidence of esophageal adenocarcinoma might be explained by synergies in the multiple mechanisms by which obesity might promote this cancer (mechanical plus humoral). The potential roles for adipokines and other circulating factors in the development of $\mathrm{BE}$ and esophageal adenocarcinoma are just beginning to be explored. Here, I summarize the currently available data for some of the leading factors under consideration. 


\section{Diabetes and insulin resistance}

Increased visceral fat is associated with insulin resistance. Insulin resistance is associated with a chronic inflammatory state, ${ }^{17}$ and elevated insulin levels stimulate cell growth and inhibit apoptosis. ${ }^{4}$ Hyperinsulinemia has been associated with the development of cancers of the colon, pancreas, and endometrium. ${ }^{18}$ In a case-control study utilizing national Veterans Health Administration data, we found no evidence for an association between diabetes mellitus with a combined outcome of adenocarcinoma of the lower esophagus or gastric cardia. ${ }^{19}$ However, we were not able to examine the effects on the risk of adenocarcinoma of the esophagus separately from the risk of gastric cardia in that study. Since then, diabetes was associated specifically with EAC in a population-based study. ${ }^{20}$ However, the effect of diabetes appeared to be partially attenuated by GERD symptoms, tobacco use, and BMI. Given the body of evidence supporting a role of insulin resistance in other epithelial cancers, further research on its role in $\mathrm{BE}$ is warranted.

\section{Adiponectin}

Adiponectin is a peptide secreted primarily by visceral adipocytes. Circulating levels are paradoxically inversely related to obesity, and are lower in men than women. Low levels have been associated with increased risk of cancers of the stomach, colon, prostate, breast, and uterus..$^{21}$ Receptors specific for adiponectin are found in the esophageal mucosa, and adiponectin can induce apoptosis and inhibit proliferation in cell lines of esophageal adenocarcinoma. ${ }^{22,23}$ In a pilot study, we found a trend toward lower blood levels of adiponectin in patients with BE compared to patients undergoing upper endoscopy for other indications. ${ }^{24}$

However, in a separate, larger study, we found no such association with total adiponectin levels. ${ }^{25}$ Adiponectin circulates in various multimers, each with specific actions. The low molecular weight (LMW) form appears to be antiinflammatory, and the high molecular weight form is proinflammatory. We found that a high ratio of LMW to total adiponectin was strongly inversely associated with the presence of $\mathrm{BE}$, compared to GERD controls, including after adjustment for abdominal obesity. ${ }^{25}$ The specific roles of the molecular weight isoforms of adiponectin in the development of BE deserve further study.

\section{Leptin}

Leptin is a peptide secreted by primarily by adipocytes; it acts to signal satiety to the brain. Most obese humans are resistant to this signal, and have elevated circulating leptin levels. In addition to its effects on satiety, leptin stimulates proliferation and inhibits apoptosis in cell lines of esophageal adenocarcinoma. ${ }^{26}$ Blood levels have been correlated with an increased risk of colorectal cancer. ${ }^{27}$ Elevated leptin has also been directly associated with the presence of BE among men, including after adjustment for GERD and obesity. ${ }^{9}$ However, an inverse association with $\mathrm{BE}$ was found in women. The reason for these discordant findings is unclear. In a smaller study composed mostly of men, no association was found between plasma leptin and BE. ${ }^{28}$ $\mathrm{BE}$ was associated with elevated gastric fundus levels of leptin, controlling for BMI, compared to patients with esophagitis or to patients undergoing upper endoscopy for other indications. Therefore, intralumenal leptin, rather than circulating leptin, may be more important for its effect on esophageal mucosa.

\section{Ghrelin}

Ghrelin is a peptide secreted by the gastric fundus. It signals to the brain to stimulate appetite. Most obese humans have suppressed circulating levels of ghrelin-that is, they continue to eat despite low levels. In a cell line of esophageal adenocarcinoma, ghrelin inhibited COX- 2 and IL- $1 \beta$ expression induced by TNF- $\alpha .{ }^{22} \mathrm{~A}$ small study found that very elevated levels of circulating ghrelin $(>3.2 \mathrm{ng} / \mathrm{mL})$ were protective against the development of esophageal adenocarcinoma, but only among overweight subjects. $^{29}$ The investigators noted a threshold effect, with no effect at lower concentrations.

\section{Insulin-like growth factor axis}

In addition to its insulin-like effects via the insulin receptor, insulin-like growth factor one (IGF1) stimulates proliferation and inhibits apoptosis in many tissues via the IGF-1 receptor, including in esophageal epithelium and intestinal crypts. ${ }^{30-33}$ Insulin enhances the synthesis of IGF-1, and hyperinsulinemia and obesity are associated with increased levels of free IGF- $1^{18,34}$ Most circulating IGF-1 is bound to IGF binding protein-3 (IGFBP3 ), and bound IGF-1 is inactive. ${ }^{35}$ IGFBP-3 inhibits cell growth and is proapoptotic. ${ }^{36}$ IGF functions primarily in paracrine and autocrine manners, 
making it difficult to study epidemiologically. However, polymorphisms of the genes encoding IGFs and the IGF receptors have been associated the risk of BE and esophageal adenocarcinoma. ${ }^{37-39}$ Elevated circulating IGF-1 levels have been associated with esophageal adenocarcinoma. ${ }^{40}$

\section{Conclusions}

Alterations in levels of circulating factors related to obesity, including adipokines directly secreted from adipose tissue, are likely involved in the development of BE and neoplastic progression. Research in this field is relatively new, and many questions remain unanswered.

\section{May body fat distribution in men, where visceral obesity is more common, be an explanation for the high incidence of $B E$ in this population?}

\section{Hashem El-Serag \\ hasheme@bcm.tmc.edu}

There are gender-related differences in the distribution of GERD and its potential complications including BE. GERD symptoms seem to be equally prevalent between men and women; erosive esophagitis is more common among men, ${ }^{41} \mathrm{BE}$ is much more common in men (70-80\%) ${ }^{42}$ whereas esophageal adenocarcinoma is dominated by men ${ }^{43}$ (Fig. 1).

Differences in obesity do not explain gender differences. Abdominal obesity explains some of the

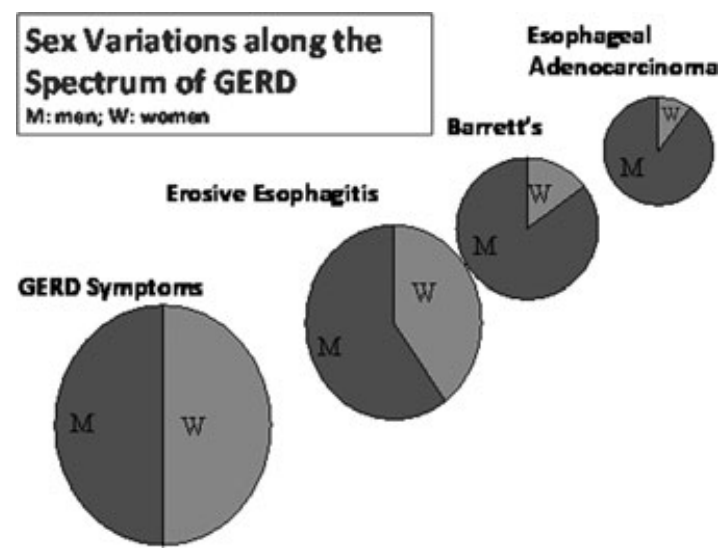

Figure 1. GERD symptoms seem to be equally prevalent between men and women, whereas erosive esophagitis is more common in men, ${ }^{41} \mathrm{BE}$ is much more common in men (70$80 \%),{ }^{42}$ and esophageal adenocarcinoma is dominated by men. epidemiological features of BE and esophageal adenocarcinoma. The distribution of body fat tends to be more visceral than truncal in high-risk groups for BO including Caucasians (compared with African Americans), and men (compared with women). ${ }^{44}$ Two recent case-control studies have shown abdominal diameter measured as WC to be a risk factor of $\mathrm{BE}$ independent of $\mathrm{BMI}$, whereas the association between BMI and BE disappeared after adjustment of abdominal diameter. ${ }^{6,45}$ These studies indicate that abdominal fat is possibly the key factor linking obesity and BE.

Only one study reported the association between WC and the risk of BE in men and women separately. Edelstein et al. in a case-control study (193 BE cases and 211 non-BE controls) conducted in the United States reported higher risk estimates for large WC and BE in men with an odds ratio of 5.9 (95\% confidence intervals 1.8-19.4) than in women with an odds ratio of 2.9 (95\% confidence intervals 8-11.0). ${ }^{6}$ The other three studies that examined the association between obesity and $\mathrm{BE}$ risk did not convey useful information on the differences between men and women. One study was conducted exclusively in men, ${ }^{46}$ the second had no abdominal obesity measurements, ${ }^{47}$ and third matched case and controls on gender. ${ }^{45}$

The leading hypothesis is that abdominal obesity promotes gastroesophageal reflux through increased pressure stress and anatomical disruption of the gastroesophageal junction.

Obese individuals may experience extrinsic gastric compression by surrounding adipose tissue leading to increase intragastric pressures and subsequent relaxation of the lower esophageal sphincter, thus facilitating abnormal reflux (Fig. 2). A study of 285 patients $^{7}$ analyzed the relationship between obesity, pressure stresses on the OGJ, and the morphology of the OGJ pressure segment itself using a solid-state manometric assembly with 36 circumferential sensors spaced at $1 \mathrm{~cm}$ intervals. Intragastric pressure, as well as gastroesophageal pressure gradient, during both expiration and inspiration was significantly higher in obese and overweight patients compared with those with a normal BMI. WC was an independent risk factor for increased intragastric pressure, whereas BMI was no longer significantly associated with pressure independent of waist. The associations were stronger in men than women. These changes lead to increased reflux across 


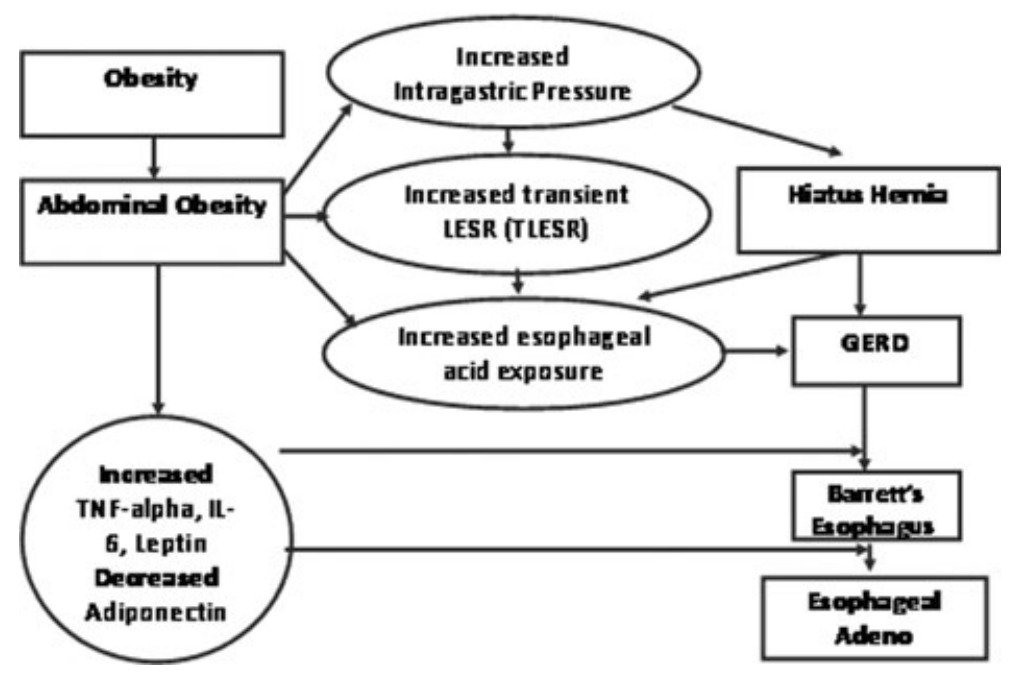

Figure 2. The relationship between obesity, pressure stresses on the OGJ, and reflux disease.

the lower esophageal sphincter with subsequent increased esophageal exposure to gastroduodenal contents. We conducted a cross-sectional study of 206 consecutive patients undergoing 24-hour pH-metry who were not on acid-suppressing medications. ${ }^{13}$ Both BMI $>30$ and high WC were associated with a significant increase in acid reflux episodes, long reflux episodes ( $>5 \mathrm{~min}$ ), time with $\mathrm{pH}<4$, as well as a calculated summary score. The association between obesity and esophageal acid exposure also became attenuated when adjusted for WC suggesting that the latter may mediate a large part of the effect of obesity. Finally, visceral fat is metabolically active, and has been strongly associated with low serum levels of potentially protective (e.g., adiponectin), or proinflammatory cytokines (e.g., interleukin-1 $\beta$, interleukin-6, and tumor necrosis factor- $\alpha$ ), which may play a role in the development of BE.

\section{Does BMI induce a difference in healing erosive esophagitis or Barrett's epithelium?}

Brian C. Jacobson

brian.jacobson@bmc.org

The short answer is "no." We generally do not monitor the natural healing rate of erosive esophagitis, and this has certainly not been correlated with BMI. Healing rates have been monitored in the set- ting of clinical trials evaluating the efficacy of acidmediating agents. However, these studies were never primarily designed to specifically look for an association between BMI and healing rates. Another, perhaps more fruitful, way to think about this question is to ask whether BMI is associated with the efficacy of acid-mediating agents. For example, one can think about whether excess body fat can affect the pharmacokinetics of these drugs. However, despite theoretical reasons why BMI might have some association with the distribution or metabolism of acid-mediating agents, there is no published evidence suggesting any important differences in the pharmacokinetics of acid-mediating agents and BMI? ${ }^{48}$

Despite this, have there been well-established clinical differences observed in patient response rates to proton pump inhibitors (PPIs) or histamine type 2 receptor antagonists (H2RAs) based upon $\mathrm{BMI}$ ? Again, the answer is no. For example, in one pooled analysis with a total of 2,458 subjects from three randomized controlled trials of PPIs in nonerosive reflux disease, BMI was not predictive for patient response. ${ }^{49}$ Further, no clear association was seen in post hoc analyses of PPI studies in both erosive and nonerosive reflux disease specifically asking if response rates differed by BMI..$^{50-51}$

Therefore, although the prevalences of erosive esophagitis and $\mathrm{BE}$ are both associated with $\mathrm{BMI},{ }^{52}$ there are no compelling data to suggest that healing rates are affected by BMI. 


\section{Is it likely that intraabdominal fat is a risk marker of GERD, BE, and adenocarcinoma? What are the means by which obesity may (also) promote carcinogenesis in BE patients?}

\author{
Michele Cicala, Silvia Cocca, and \\ Michele Pier Luca Guarino \\ m.cicala@unicampus.it
}

Obesity has increased considerably in the last few decades, with a prevalence from $15 \%$ in $1976-1980$ to $32 \%$ in $2003-2004$ in the United States and in several parts of Europe and Asia. The health implications of obesity are relevant, affecting almost every apparatus. Obesity and GERD, as well as GERD complications, such as $\mathrm{BE}$ and adenocarcinoma, are clearly related. ${ }^{45,53}$

The finding that increased abdominal fat, rather than a high BMI value, is strongly associated with GERD and $\mathrm{BE}$ has been well established. In a hospital-based study, the increase in visceral, rather than subcutaneous, fat, assessed at computerized tomography (CT) scan, has been shown to be an independent and strong risk factor for BE. ${ }^{53}$ Furthermore, data from a case control study in which patients with an incidental diagnosis of $\mathrm{BE}$ were compared both with controls and patients with GERD, have provided further support to the hypothesis that abdominal obesity, in terms of abdominal circumference, is a risk factor of $\mathrm{BE}$ and is associated with severe GERD symptoms. ${ }^{2}$ Another measure of abdominal girth, the waist to hip ratio, more than the BMI, seems to be strongly associated, irrespective of symptom severity, with the presence of BE and, in particular, with long-segment Barrett's metaplasia. ${ }^{6}$

A positive association between increased abdominal diameter and the risk of esophageal adenocarcinoma, but not cardia or squamous cell carcinoma, has recently been demonstrated by Kubo et al. ${ }^{54}$ These findings might explain the higher risk of $\mathrm{BE}$ and adenocarcinoma in white men, who are more commonly affected by abdominal obesity.

A potential limitation of all case-control studies is the difficulty in establishing a temporal association between exposure and outcome, for instance between the onset and duration of obesity and the endpoint, the disease. Moreover, observational studies are subject to several other confounding factors, and the effect of weight loss on the development of the disease has not yet been established.
In summary, given all these limitations, intraabdominal fat could likely be considered a risk marker of GERD, BE and adenocarcinoma; however, further research is mandatory to obtain direct evidence of this pathway.

Another important question concerns the means by which obesity may also promote carcinogenesis in BE patients. It is known that metabolic effects of obesity, particularly abdominal obesity, may also contribute to carcinogenesis. Fat, located in the visceral compartment, induces changes in hormone production such as adiponectin and leptin, insulin and IGFs, and in cytokine production, all of which are able to affect cellular proliferation and apoptosis. ${ }^{55}$ Several studies, have demonstrated that increased serum levels of leptin, a cytokine discovered in 1994 as a regulator of body weight and energy balance, are directly correlated with onset of various tumors and with an increased risk of BE. ${ }^{55}$ On the other hand, levels of adiponectin, that are usually inversely associated with the risk of malignancies related to obesity, are found to be lower in obese patients than in normal weight patients. ${ }^{55}$ As a result of this impaired metabolic activity the increased production of fatty acids, leading to insulin resistance and result in hyperinsulinemia, may have a direct effect upon insulin receptors as well as an effect mediated by decreasing IGF binding proteins, thus increasing IGF bioavailability, both of which result in reduced apoptosis and increased cell proliferation. Finally, abdominal obesity could also play a role in the development of BE by increasing intragastric pressure and, therefore, promoting reflux. Surprisingly, evidence confirming this pathway is still weak. ${ }^{53}$

Further investigations on the endocrine effects of adipose tissue and its potential role in carcinogenesis are now mandatory.

\section{Does surgery for obesity result in better control of GERD and risk of malignancy in BE?}

\section{Reza A. Hejazi and Richard W. McCallum} richard.mccallum@ttuhsc.edu

The prevalence of GERD is markedly higher in overweight and obese individuals as compared to those with normal BMI, ${ }^{56}$ since GERD itself is now recognized as an obesity-related comorbidity. 

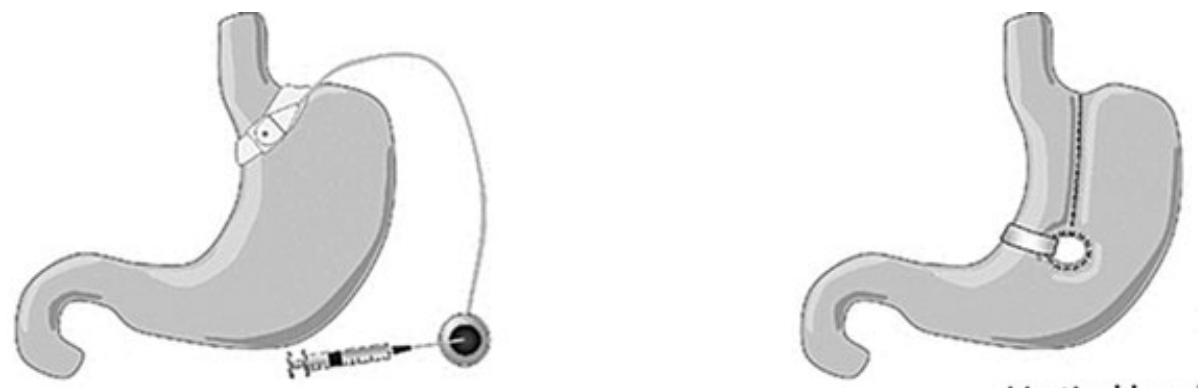

Adjustable laparocopic gastric banding

Vertical bending gastroplasty

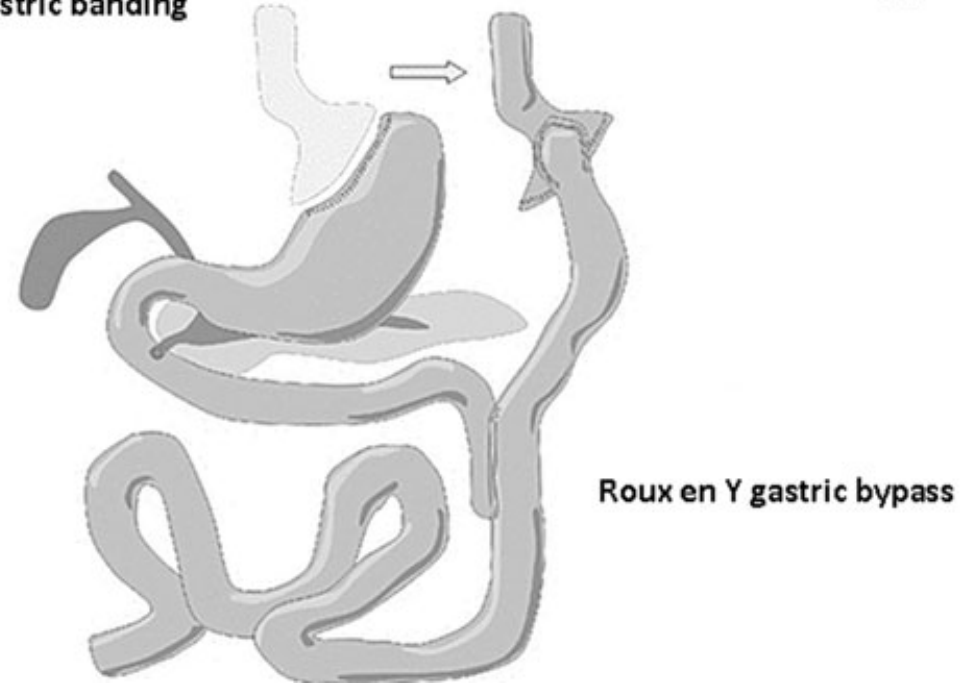

Figure 3. Adjustable laparoscopic gastric banding; Vertical banding gastroplasty; Roux en Y gastric bypass. Adapted from De Groot et al. ${ }^{58}$ and with permission from John Wiley and Sons.

Bariatric surgery actually has become a widely accepted form of treatment for severe obesity, and several studies have demonstrated a significant reduction in GERD symptoms and medication utilization, as well as weight and metabolic comorbidity, including diabetes, hypertension and dyslipidemia. ${ }^{57}$ The different techniques for performing bariatric surgery have variable effects on GERD and subsequently on the risk of BE. Roux en $\mathrm{Y}$ gastric bypass (Fig. 3), as the most performed bariatric operation in the United States, was found in majority of studies to have a positive effect on GERD symptoms. ${ }^{3}$ Vertical banding gastroplasty as a restrictive procedure, on the other hand, has no change or even, based on some reports, may cause an increase of GERD. ${ }^{58}$ Although laparoscopic adjustable gastric banding might have a beneficial effect, the results of this procedure are still conflicting. ${ }^{3}$ Preoperative selection of GERD patients, as well as em- ploying the most beneficial procedure, are the goals. The good news here is that the resolution of Barrett's disease and atypia of the esophageal epithelial after gastric bypass and laparoscopic gastric bypass/LAPBAND were reported in just two cases. ${ }^{59}$ After laparoscopic placement of adjustable gastric banding, Barrett's esophagus could be a rare (one case) but not unexpected complication, and the incidence of it is still unknown. ${ }^{60}$

\section{Conclusions}

GERD is common problem in obese and overweight populations. Surgical treatment of obesity has positive effect on GERD and its complications, such as BE.

Roux en $\mathrm{Y}$ gastric bypass has the most favorable impact on GERD symptoms, although certain restrictive procedures such as laparoscopic gastric bypass/LAP-BAND have also been reported to 
have some postive effects on Barrett's disease and esophageal dysplasia.

\section{What is the role of double-contrast esophagography in the diagnosis and screening for $B E$ in patients with reflux symptoms?}

Marc S. Levine

marc.levine@uphs.upenn.edu

The classic radiologic features of $\mathrm{BE}$ consist of a mid-esophageal stricture or ulcer, often associated with a sliding hiatal hernia and GER. ${ }^{61}$ These strictures typically appear as ring-like constrictions or as tapered areas of narrowing below the level of the aortic arch. However, strictures are actually more common in the distal esophagus in patients with $\mathrm{BE}$, so most cases do not fit the classic description of a high stricture or ulcer. ${ }^{62}$ A reticular mucosal pattern has also been described as a relatively specific sign of BE on double-contrast esophagograms, particularly if located just distal to a midesophageal stricture. ${ }^{62}$ However, a reticular mucosal pattern is present on barium studies in only $5-30 \%$ of all patients with BE.

Other morphologic findings of reflux disease, such as hiatal hernias, GER, reflux esophagitis, and peptic strictures, can be detected on double-contrast studies in the vast majority of patients with $\mathrm{BE}$, but these findings frequently occur in patients with uncomplicated reflux disease. ${ }^{63}$ Thus, those radiographic findings that are relatively specific for $\mathrm{BE}$ are not sensitive, and those findings that are more sensitive are not specific. As a result, double-contrast esophagography has traditionally been thought to have limited value for diagnosing BE.

Gilchrist et al. introduced a novel approach for the diagnosis of BE on double-contrast esophagography by stratifying patients based on specific radiologic criteria. ${ }^{64}$ Patients who were classified at high risk for $\mathrm{BE}$ because of a high stricture or ulcer or a reticular mucosal pattern were almost always found to have this condition, so endoscopy and biopsy should be performed in this group for a definitive diagnosis. A larger group of patients were classified at moderate risk for BE because of esophagitis or strictures in the distal esophagus; $16 \%$ of these patients were found to have BE, so the decision for endoscopy in this group should be based on the severity of symptoms, age, and overall health of the patients (i.e., whether they are reasonable candidates for surveillance). However, the majority of patients were classified as low risk for $\mathrm{BE}$ because of the absence of esophagitis or strictures in the esophagus; many of these patients were found to have mild reflux esophagitis, but less than $1 \%$ had BE, so these patients can be treated empirically for their reflux symptoms without need for endoscopy. Thus, the major value of the barium study in patients with reflux symptoms is its ability to stratify them into these various risk groups for $\mathrm{BE}$ to determine the relative need for endoscopy and biopsy. ${ }^{64,65}$

\section{Cross-sectional imaging in the detection of $B E$}

Richard M. Gore, Kiran H. Thakrar, Geraldine M. Newmark, Uday K. Mehta, and Jonathan W. Berlin rgore@uchicago.edu

Upper gastrointestinal endoscopy and barium esophagography are the primary means of evaluating patients with known or suspected esophageal pathology. ${ }^{65,66}$ Although these examinations superbly depict the esophageal mucosa, they are limited by their inability to directly image the esophageal wall and surrounding adventitia, fat, and lymph nodes. Recent advances in MDCT now allow routine visualization of the entire esophagus in a single breath-hold with thin collimation and isotropic voxels. This allows the production of high-quality multiplanar reformations and $3 \mathrm{D}$ reconstruction images. The sensitivity of MDCT in the evaluation of esophageal disease is further enhanced by proper distention of the esophagus by the oral administration of effervescent granules and water and optimally timed administration of intravenous contrast material.

Although detection of the actual metaplastic columnar epithelium of $\mathrm{BE}$ is beneath the spatial resolution of MDCT, there are a number of secondary features that help suggest diagnosis, especially of long segment, circumferential disease.

MDCT is superb in the identification of hiatal hernias (Fig. 4). There is extension of a portion of the proximal stomach or other abdominal contents into the lower mediastinum. An abnormally wide 
A

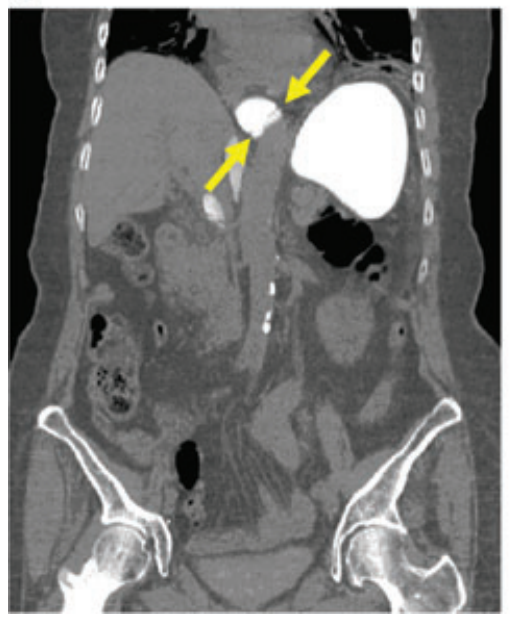

B

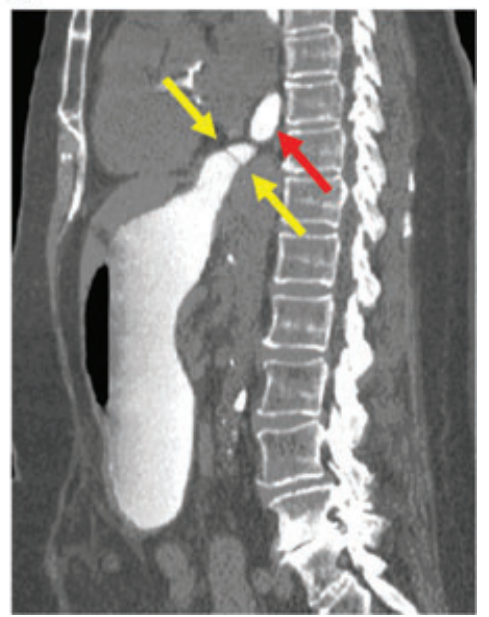

Figure 4. MDCT scan in a patient subsequently proven to have Barrett's esophagus. (A) Coronal and (B) sagittal reformatted images demonstrate a hiatal hernia and Schatzki's ring (yellow arrows). Note the gastroesophageal reflux (red arrow).

esophageal hiatus with increased separation of the esophagus and diaphragmatic crura is often present.

MDCT can also document the presence of gastroesophageal reflux (see Fig. 4) however this is less reliably detected than by conventional barium esophagrams and nuclear medicine reflux studies. Oral contrast material is routinely given for abdominal and pelvic CT scans and this contrast material may be visualized in the distal esophagus and can show the presence of a stricture or Schatzki's ring.

MDCT is superb in depicting mural thickening (Fig. 5) of the esophagus and the diagnosis can be confidently made in patients with a well-distended esophagus. Mural thickening of the esophagus as shown on MDCT is nonspecific and can be seen in a variety of benign and malignant disorders including: reflux esophagitis, infectious esophagitis, tuberculosis, radiation esophagitis, eosinophilic esophagitis, BE, Crohn's disease of the esophagus, achalasia, scleroderma, diffuse esophageal spasm, esophageal pseudodiverticulosis, adenocarcinoma of the esophagus, squamous cell carcinoma of the esophagus, lymphoma of the esophagus, and esophageal metastases. ${ }^{67}$

Five $\mathrm{mm}$ is a useful threshold for esophageal wall thickening in patients with esophagitis. Indeed, some $55 \%$ of all patients with esophagitis have an esophageal wall thickness of $5 \mathrm{~mm}$ or greater on MDCT. Benign or malignant tumors of the esopha- gus are usually manifested on CT by focal, asymmetric thickening of the esophageal wall, whereas mural thickening in esophagitis is concentric and circumferential, and usually involves a relatively long segment of the esophagus. ${ }^{67}$

Some $20 \%$ of patients with esophagitis may also demonstrate mural stratification, in which the axially imaged esophagus has a target appearance. This is caused by the combination of mucosal enhancement and a hypodense submucosa. In the small bowel and colon, the target sign almost always indicates benign disease associated with submucosal edema resulting from inflammation, infection, or ischemia.

Other clues to esophageal pathology include the distribution of intraluminal air on MDCT. Intraluminal air is normal in the esophagus, but distention of more than $10 \mathrm{~mm}$ at a fixed point, such as the carina, is uncommon. Any segment over $20 \mathrm{~mm}$ should be considered abnormal. The normal lower esophageal sphincter should be closed. In the upper $30 \%$ of the esophagus (to about the top of the aortic arch) and from $61 \%$ to $75 \%$ of the length of the esophagus (behind the base of the heart) less than $5 \%$ of the lumina are larger than $10 \mathrm{~mm}$ in diameter. ${ }^{68}$

The greatest luminal dimensions and variation occur in the area between where the diaphragm first appears and where the esophagus enters the abdomen. Here, $15 \mathrm{~mm}$ should the upper limits 
A

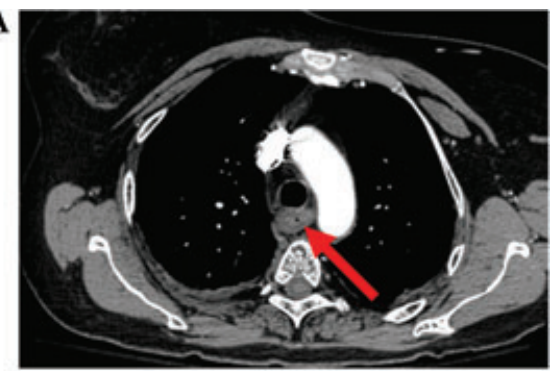

B

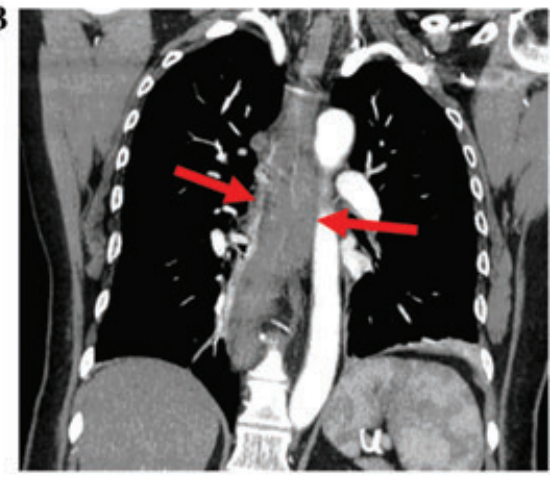

C

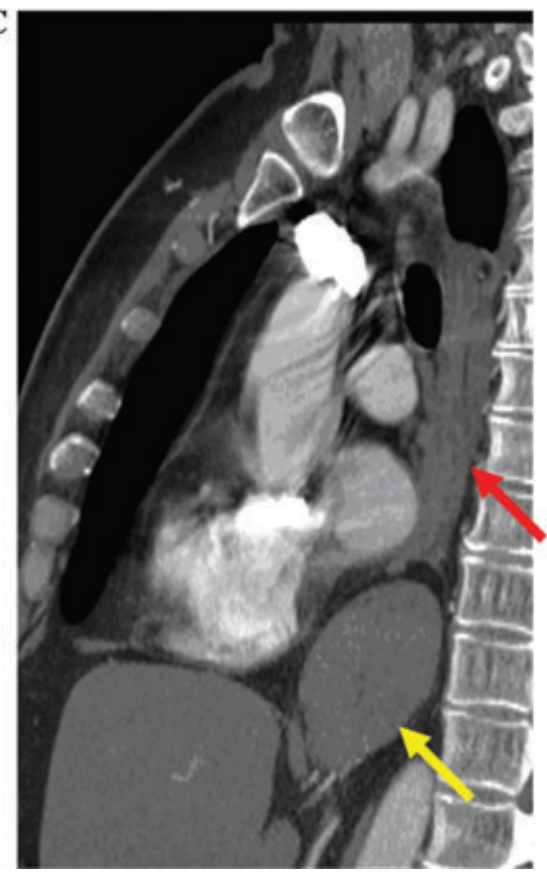

Figure 5. MDCT scan in a patient with reflux esophagitis and a long segment of Barrett's esophagus proven by endoscopic biopsy. (A) Axial, (B) coronal reformatted, and (C) sagittal reformatted images show a large hiatal hernia (yellow arrows) associated with a long segment of mural thickening of the esophagus (red arrow). (A) Submucosal edema produces the target appearance of the axially imaged esophagus. of normal. An air-fluid level on any section of the esophagus is abnormal. ${ }^{68}$

In summary, the following constellation of findings should raise the possibility of $\mathrm{BE}$ or at least esophagitis on MDCT: hiatal hernia, mural thickening of the esophagus in a symmetric, circumferential manner, the target sign, too much intraluminal air, air-fluid levels, foodstuffs or tablets in the esophagus. These CT findings should be carefully searched for on pulmonary embolism and thoracic aortic dissection MDCT scans performed on patients with chest discomfort. A minority number of these patients will ultimately be shown to have significant esophageal pathology.

\section{Can PET/CT differentiate BE from other benign esophageal disorders?}

Richard M. Gore, Kiran H. Thakrar, Geraldine M. Newmark, Uday K. Mehta, and Jonathan W. Berlin rgore@uchicago.edu

Combined PET/CT with fluorine ${ }^{18}$ fluorodeoxyglucose (FDG) is a hybrid device that fuses structural information provided by multidetector CT with the functional imaging provided by PET (Fig. 6). This technique improves the radiologic assessment of normal anatomic structures and pathological lesions. Cellular FDG uptake is predominantly related to expression of the protein glucose transport 1 . This protein is ubiquitously expressed in almost all cell types, but its over expression in dysplastic and malignant tissue is quite frequent and leads to intracellular accumulation of FDG, which is visualized on PET. The high lesion to background contrast and whole body data acquisition on FDG PET represent critical advantages over CT and MRI, where contrast between pathologic and normal structures may be limited. FDG PET has been developed to quantitatively assess local glucose metabolism. PET can help differentiate benign and malignant tumors, determine the degree of malignancy, evaluate the effectiveness of chemotherapy and/or radiotherapy, and help predict prognosis. Indeed FDG PET has been used to screen for malignancies. ${ }^{69}$

FDG PET is a well-accepted method for the detection and staging of a number of malignancies including lung, breast, colorectal, and esophageal 


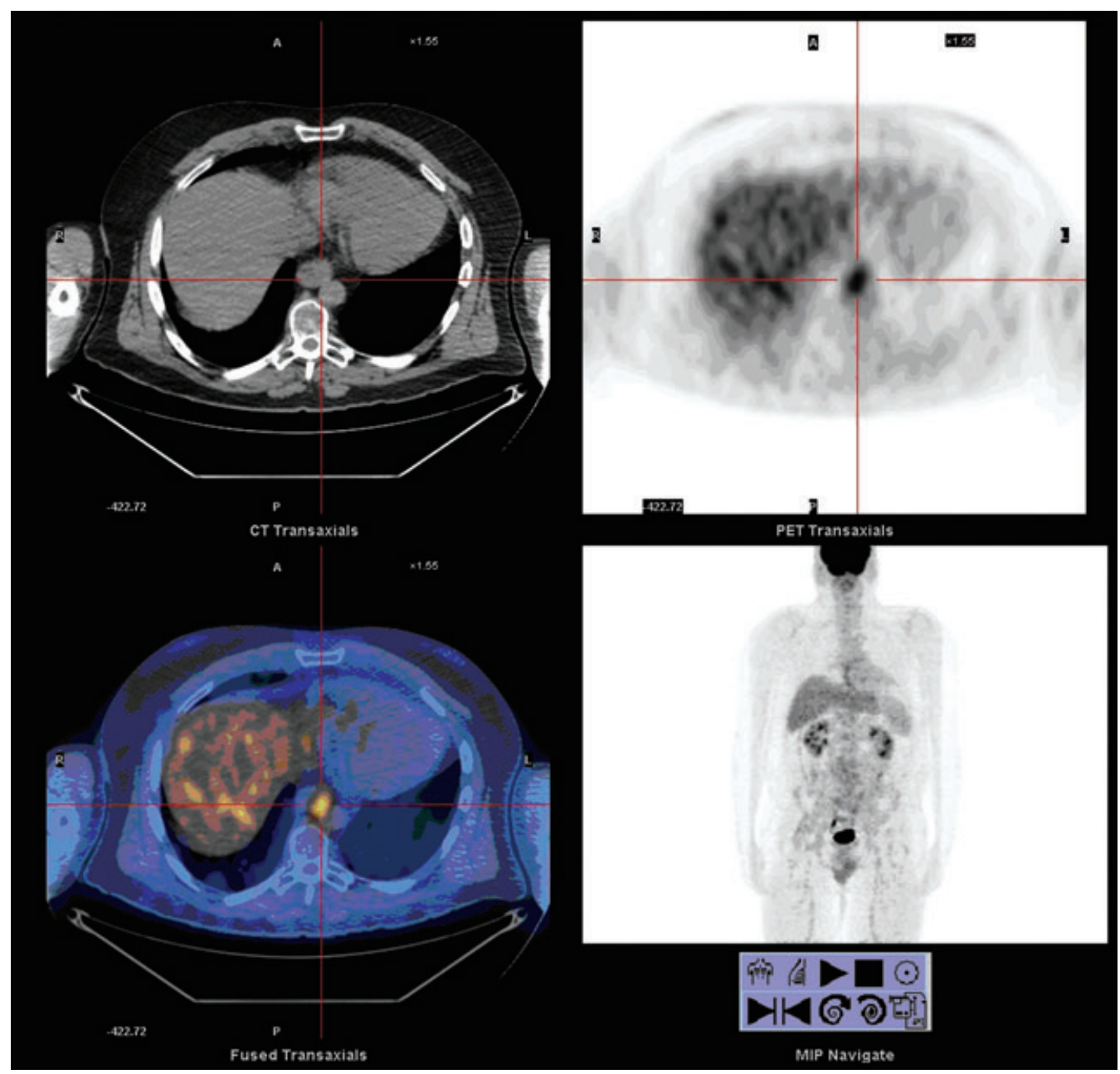

Figure 6. PET/CT of Barrett's esophagus. (A) Axial, (B) coronal, and (C) sagittal images of the esophagus show mural thickening of the esophagus on the CT (white arrow), increased metabolic activity on PET (red arrow), mural thickening of the esophagus and increased metabolic activity on the PET/CT (yellow arrow), and increased metabolic activity on coronal scanogram (black arrow) components of the examination.

cancer. The applications of FDG PET in the clinical diagnosis of BE have not been established.

Preliminary experimental work has demonstrated the efficacy of high-resolution PET scanning to examine the degree and time dependency of changes in FDG uptake in rat esophageal tissues during the esophageal reflux injury carcinogenic progression pathway. FDG accumulation is significantly elevated in esophageal tissues three and six month (correlating to BE with high grade dysplasia and early adenocarcinoma) than that at one week and one month (reflux esophagitis), and this accumulation corresponds to the histopathologically observed progression of squamous epithelium to hyperplasia to metaplasia and onto adenocarcinoma. This early work suggests that FDG PET scanning may play an important role in the assessment of a subset of patients that are at risk for the esophagitis to metaplasia to dysplasia to esophagitis-adenocarcinoma sequence. $^{70}$

Nonspecific esophageal uptake is a common finding when interpreting PET/CT studies and is usually due to reflux esophagitis. This is not surprising considering that about $15 \%$ of the population suffers from reflux disease. These benign lesions, 


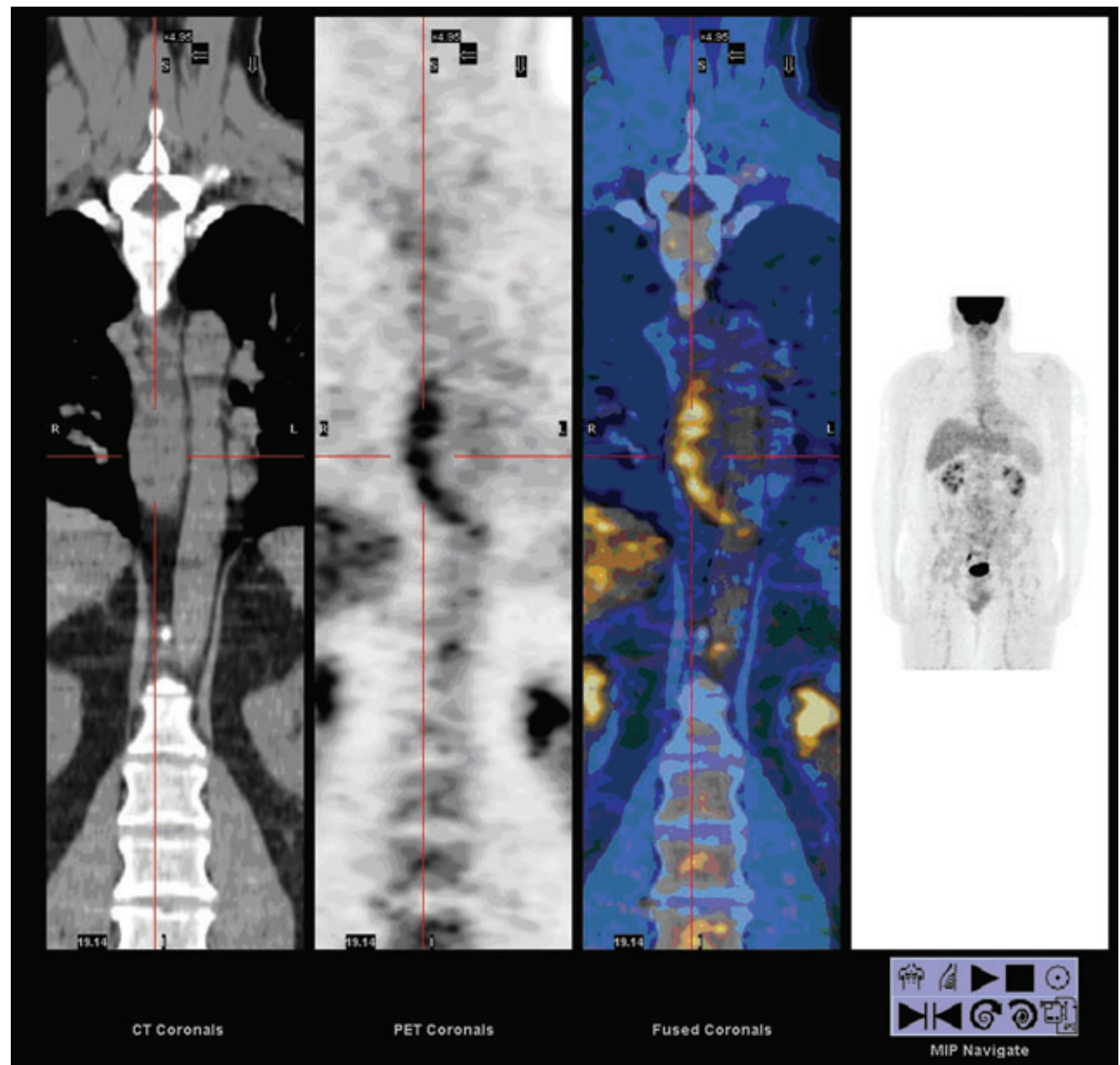

Figure 6. Continued

however, can resemble early esophageal malignancies. The clinical challenge is that, on the one hand, the only chance for cure of esophageal carcinoma is its early detection, and, on the other hand, the rate of false positives among nonspecific esophageal uptake is too high to recommend further evaluation with endoscopy in all of those cases. Therefore, the differentiation between early malignant and benign lesions has important clinical implications. ${ }^{71,72}$

The differential diagnosis of increased FDG uptake on PET CT includes: reflux esophagitis, infectious esophagitis, radiation esophagitis, BE, primary and secondary achalasia, scleroderma, diffuse esophageal spasm, intramural pseudodiverticulosis, tuberculosis, Crohn's disease, adenocarci- noma, squamous cell carcinoma, lymphoma, and metastases.

When evaluating abnormal PET CT findings of the esophagus, Roedl et al. ${ }^{73}$ have suggested closely interrogating the scans for the following features: esophageal thickness, focality and location of the lesion, eccentricity of the esophageal thickening, and degree of metabolic activity-standard uptake value. Roedl et al. found no significant differences in esophageal thickness on CT and location of the lesion between the 36 early malignant and the 66 benign lesions. However, higher SUV, greater lesion focality, and greater eccentricity of the mural thickening are significantly increased in the early malignant group when compared with benign lesions. $^{73}$ 


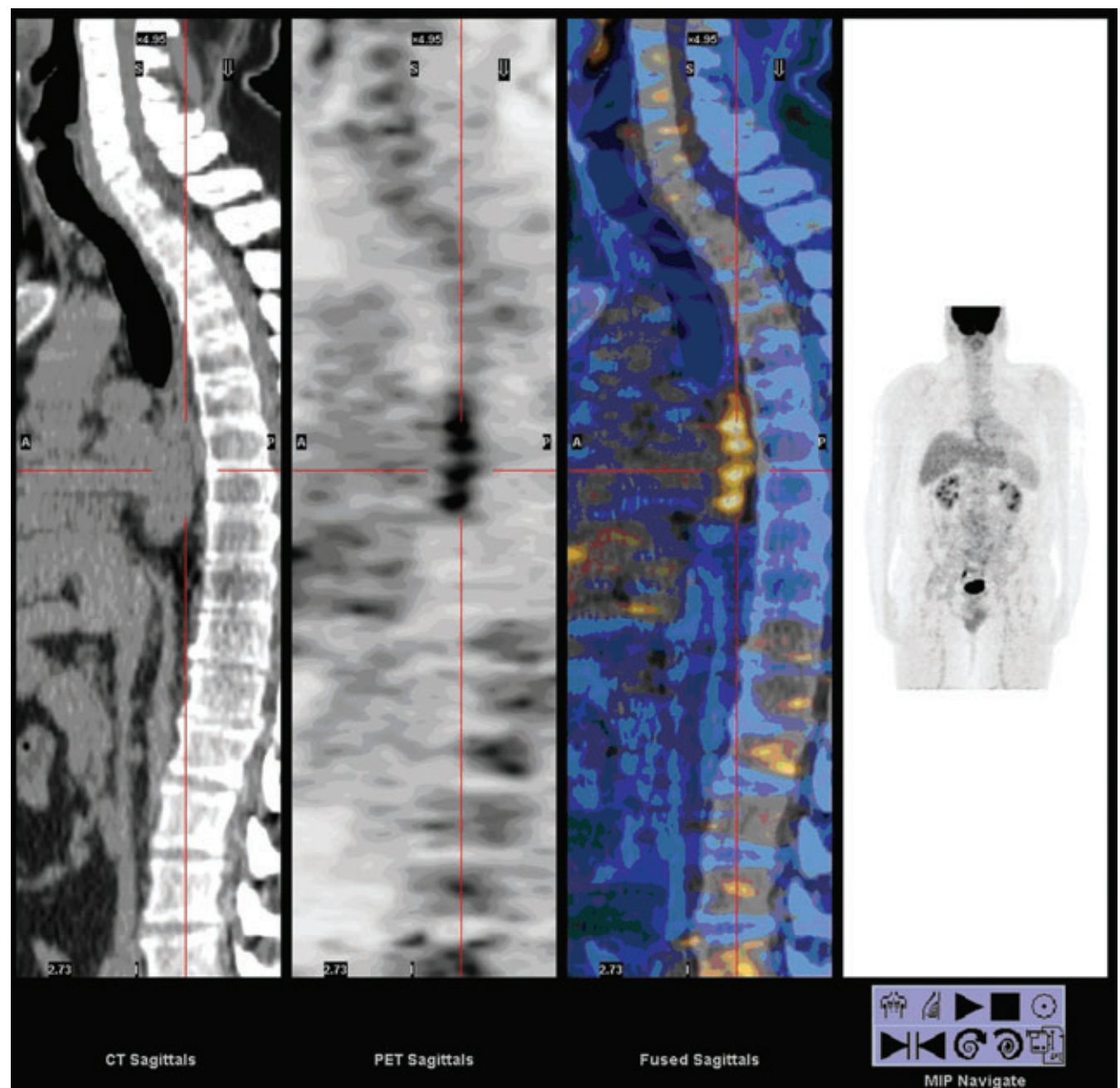

Figure 6. Continued

At the present time, PET CT is not sufficiently sensitive and specific enough to differentiate BE from other benign disorders but, with technical improvements, may help detect malignant transformation and even dysplasia in patients with this disorder.

\section{Conflicts of interest}

The authors declare no conflicts of interest.

\section{References}

1. Quera, R., K. O'Sullivan \& E.M. Quigley. 2006. Surveillance in Barrett's oesophagus: will a strategy focused on a high-risk group reduce mortality from oesophageal adenocarcinoma? Endoscopy 38: 162-169.

2. Chak, A., T. Lee, M.F. Kinnard, et al. 2002. Familial aggregation of Barrett's oesophagus, oesophageal adenocarcinoma, and oesophagogastric junctional adenocarcinoma in Caucasian adults. Gut. 51: 323-328.

3. Taylor, J.B. \& J.H. Rubenstein. 2010. Meta-analyses of the effect of symptoms of gastroesophageal reflux on the risk of Barrett's esophagus. Am. J. Gastroenterol. 105: 17301737.

4. Prasad, G.A., A. Bansal, P. Sharma \& K.K. Wang. 2010. Predictors of progression in Barrett's esophagus: current knowledge and future directions. Am. J. Gastroenterol. 105: 14901502.

5. Lagergren, J., R. Bergström \& O. Nyrén. 1999. Association between body mass and adenocarcinoma of the esophagus and gastric cardia. Ann. Intern. Med. 130: 883890.

6. Edelstein, Z. et al. 2007. Central adiposity and risk of Barrett's esophagus. Gastroenterology 133: 403-411.

7. Pandolfino, J. et al. 2006. Obesity: a challenge to esophagogastric junction integrity. Gastroenterology 130: 639-649. 
8. Wu, J. et al. 2007. Obesity in associated with increased transient lower esophageal sphincter relaxation. Gastroenterology 132: 883-889.

9. Kendall, B.J. et al. 2008. Leptin and the risk of Barrett's oesophagus. Gut. 57: 448-454.

10. Jacobson, B. et al. 2008. Postmenopausal hormone use and symptoms of gastroesophageal reflux. Arch. Intern. Med. 168: 1-7.

11. Hampel, H., N.S. Abraham \& H.B. El-Serag. 2005. Metaanalysis: obesity and the risk for gastroesophageal reflux disease and its complications. Ann. Intern. Med. 143: 199211.

12. Jacobson, B.C., S.C. Somers, C.S. Fuchs, et al. 2006. Bodymass index and symptoms of gastroesophageal reflux in women. N. Engl. J. Med. 354: 2340-2348.

13. El-Serag, H.B., G.A. Ergun, J. Pandolfino, et al. 2007. Obesity increases oesophageal acid exposure Gut. 56: 749-755.

14. Edelstein, Z.R., M.P. Bronner, S.N. Rosen \& T.L. Vaughan. 2009. Risk factors for Barrett's esophagus among patients with gastroesophageal reflux disease: a community clinicbased case-control study. Am. J. Gastroenterol. 104: 834-842.

15. Mohamed-Ali, V., S. Goodrick, A. Rawesh, et al. 1997. Subcutaneous adipose tissue releases interleukin-6, but not tumor necrosis factor-alpha, in vivo. J. Clin. Endocrinol. Metab. 82: 4196-4200.

16. Fried S.K., D.A. Bunkin \& A.S. Greenberg. 1998. Omental and subcutaneous adipose tissues of obese subjects release interleukin-6: depot difference and regulation by glucocorticoid. J. Clin. Endocrinol. Metab. 83: 847-850.

17. Festa, A., R. D’Agostino Jr, G. Howard, et al. 2000. Chronic subclinical inflammation as part of the insulin resistance syndrome: the Insulin Resistance Atherosclerosis Study (IRAS). Circulation 102: 42-47.

18. Kaaks, R. \& A. Lukanova. 2001. Energy balance and cancer: the role of insulin and insulin-like growth factor-I. Proc. Nutr. Soc. 60: 91-106.

19. Rubenstein, J.H., J. Davis, J.A. Marrero \& J.M. Inadomi. 2005. Relationship between diabetes mellitus and adenocarcinoma of the oesophagus and gastric cardia. Aliment. Pharmacol. Ther. 22: 267-271.

20. Neale, R.E., J.D. Doecke, N. Pandeya, et al. 2009. Does type 2 diabetes influence the risk of oesophageal adenocarcinoma? Br. J. Cancer 100: 795-798.

21. Kelesidis, I., T. Kelesidis \& C.S. Mantzoros. 2006. Adiponectin and cancer: a systematic review. Br. J. Cancer 94: 1221-1225.

22. Konturek, P.C., G. Burnat, T. Rau, et al. 2008. Effect of adiponectin and ghrelin on apoptosis of barrett adenocarcinoma cell line. Dig. Dis. Sci. 53: 597-605.

23. Ogunwobi, O.O. \& I.L. Beales. 2008. Globular adiponectin, acting via adiponectin receptor-1, inhibits leptin-stimulated oesophageal adenocarcinoma cell proliferation. Mol. Cell. Endocrinol. 285: 43-50.

24. Rubenstein, J.H., A. Dahlkemper, J.Y. Kao, et al. 2008. A pilot study of the association of low plasma adiponectin and Barrett's esophagus. Am. J. Gastroenterol. 103: 1358-1364.

25. Rubenstein J.H., J.Y. Kao, R.D. Madanick, et al. 2009. Association of adiponectin multimers with Barrett's esophagus. Gut 58: 1583-1539.
26. Ogunwobi, O., G. Mutungi \& I.L. Beales. 2006. Leptin stimulates proliferation and inhibits apoptosis in Barrett's esophageal adenocarcinoma cells by cyclooxygenase-2dependent, prostaglandin-E2-mediated transactivation of the epidermal growth factor receptor and c-Jun NH2terminal kinase activation. Endocrinology 147: 45054516.

27. Stattin, P., A. Lukanova, C. Biessy, et al. 2004. Obesity and colon cancer: does leptin provide a link? Int. J. Cancer 109: 149-152.

28. Francois, F., J. Roper, A.J. Goodman, et al. 2008. The association of gastric leptin with oesophageal inflammation and metaplasia. Gut 57: 16-24.

29. de Martel, C., T.D. Haggerty, D.A. Corley, et al. 2007. Serum ghrelin levels and risk of subsequent adenocarcinoma of the esophagus. Am. J. Gastroenterol. 102: 1166-1172.

30. Vinayek, R., L.S. Pichney, U. Tantry, et al. 1994. Characterization of insulin-like growth factor I receptors in human esophageal epithelial cells. Am. J. Physiol. 267: G105-G114.

31. Jimenez, P., A. Lanas, E. Piazuelo \& F. Esteva. 1998. Effect of growth factors and prostaglandin E2 on restitution and proliferation of rabbit esophageal epithelial cells. Dig. Dis. Sci. 43: 2309-2316.

32. Tchorzewski, M.T., F.G. Qureshi, M.D. Duncan, et al. 1998. Role of insulin-like growth factor-I in esophageal mucosal healing processes. J. Lab. Clin. Med. 132: 134-141.

33. Lund, P.K. 1999. IGFs and the digestive tract. In Roberts C.T., Rosenfeld R.G., Eds.: 517-544. The IGF System: Molecular Biology, Physiology, and Clinical Applications. Totowa, NJ: Humana Press.

34. Renehan, A.G., J. Frystyk \& A. Flyvbjerg. 2006. Obesity and cancer risk: the role of the insulin-IGF axis. Trends Endocrinol. Metab. 17: 328-336.

35. Yu, H. \& T. Rohan. 2000. Role of the insulin-like growth factor family in cancer development and progression. J. Natl. Cancer Inst. 92: 1472-1489.

36. Leroith, D., W. Zumkeller \& R.C. Baxter, Eds. 2003. Insulinlike Growth Factors. Landes Bioscience/Eurekah.com; New York. Georgetown, TX.

37. Macdonald K, G.A. Porter, D.L. Guernsey, et al. 2009. A polymorphic variant of the insulin-like growth factor type I receptor gene modifies risk of obesity for esophageal adenocarcinoma. Cancer Epidemiol. 33: 37-40.

38. Hoyo, C., J.M. Schildkraut, S.K. Murphy, et al. 2009. IGF2R polymorphisms and risk of esophageal and gastric adenocarcinomas. Int. J. Cancer 125: 2673-2678.

39. McElholm, A.R., A-J McKnight, C.C. Patterson, et al. 2010. A population-based study of IGF axis polymorphisms and the esophageal inflammation, metaplasia, adenocarcinoma sequence. Gastroenterology 139: 204-12.e3.

40. Sohda, M., H. Kato, T. Miyazaki, et al. 2004. The role of insulin-like growth factor 1 and insulin- like growth factor binding protein 3 in human esophageal cancer. Anticancer Res. 24: 3029-3034.

41. El-Serag, H.B., N.J. Petersen, J. Carter, et al. 2004. Gastroesophageal reflux among different racial groups in the United States. Gastroenterology 126: 1692-1699.

42. Falk, G.W., P.N. Thota, J.E. Richter et al. 2005. Barrett's esophagus in women: demographic features and 
progression to high-grade dysplasia and cancer. Clin Gastroenterol. Hepatol. 3: 1089-1094.

43. Pohl, H. \& H.G. Welch. 2005. The role of overdiagnosis and reclassification in the marked increase of esophageal adenocarcinoma incidence. J. Natl. Cancer Inst. 97: 142146.

44. Weinsier, R.L., G.R. Hunter, B.A. Gower, et al. 2001. Body fat distribution in white and black women: different patterns of intraabdominal and subcutaneous abdominal adipose tissue utilization with weight loss. Am. J. Clin. Nutr. 74: 631-636.

45. Corley, D.A., A. Kubo, T.R. Levin, et al. 2007. Abdominal obesity and body mass index as risk factors for Barrett's esophagus. Gastroenterology 133: 34-41.

46. El-Serag, H.B., P. Kvapil, J. Hacken-Bitar, et al. 2005. Abdominal obesity and the risk of Barrett's esophagus. Am. J. Gastroenterol. 100: 2151-2156.

47. Smith, K.J., S.M. O’brien, B.M. Smithers, et al. 2005. Interactions among smoking, obesity, and symptoms of acid reflux in Barrett's esophagus. Cancer Epidemiol. Biomarkers Prev. 14(11 Pt 1): 2481-2486.

48. Jacobson, B.C. 2008. Body mass index and the efficacy of acid-mediating agents for GERD. Dig. Dis. Sci. 53: 23132317.

49. Talley, N.J. et al. 2006. Predictors of treatment response in patients with non-erosive reflux disease. Aliment Pharmacol. Ther. 24: 371-376.

50. Sharma, P. et al. 2007. Effect of Obesity on Symptom Resolution in Patients with Gastroesophageal Reflux Disease (GERD). Am. J. Gastroenterol. 102: S139-140 [abstract].

51. Vakil, N. et al. 2007. Is Obesity the Cause of Reduced Healing Rates in Advanced Grades of Erosive Esophagitis (EE)? Am. J. Gastroenterol. 102: S445.

52. Hampel, H., N. Abraham \& H. El-Serag. 2005. Meta-analysis: Obesity and the risk for astroesophageal reflux disease and its complications. Ann. Intern. Med. 143: 199-211.

53. El Serag H.E. 2008. The association between obesity and GERD: a review of the epidemiological evidence. Dig. Dis. Sci. 53: 07-12.

54. Kubo, A., D.A. Corley, et al. 2006. Body mass index and adenocarcinomas of the esophagus or gastric cardia: a systematic review and meta-analysis. Cancer Epidemiol. Biomarkers Prev. 15: 872-878.

55. Thompson, O.M., S.A.A. Beresford, Kirket E.A., et al. 2010. Serum Leptin and Adiponectin Levels and Risk of Barrett's Esophagus and Intestinal Metaplasia of the Gastroesophageal Junction. Obesity 18: 2204-2211.

56. Hampel, H., N.S. Abraham \& H.B. El-Serag. 2005. Metaanalysis: obesity and the risk for gastroesophageal reflux disease and its complications. Ann. Intern. Med. 143: 199211.

57. Buchwald, H., Y. Avidor, E. Braunwald, M.D. Jensen, et al. 2004. Bariatric surgery: a systematic review and metaanalysis. JAMA 292: 1724-1737.
58. De Groot, N.L., J.S. Burgerhart, P.C. Van De Meeberg, de D.R. Vries, et al. 2009. Systematic review: the effects of conservative and surgical treatment for obesity on gastro-oesophageal reflux disease. Aliment. Pharmacol. Ther. 30: 1091-1102.

59. Chang, C.G. \& E. Perez. 2009. Case reports-resolution of Barrett's disease and esophageal epithelial atypia after gastric bypass and LAP-BAND. Obes. Surg. 19: 1597-1598.

60. Varela, J.E. 2010. Barrett's esophagus: a late complication of laparoscopic adjustable gastric banding. Obes. Surg. 20: 244-246.

61. Robbins, A.H., J.A. Hermos, E.M. Schimmel, et al. 1977. The columnar-lined esophagus: analysis of 26 cases. Radiology 123: $1-7$.

62. Levine M.S., H.Y. Kressel, D.F. Caroline, et al. 1983. Barrett esophagus: reticular pattern of the mucosa. Radiology 147: 663-667.

63. Chen, Y.M., D.W. Gelfand, D.J. Ott, et al. 1985. Barrett esophagus as an extension of severe esophagitis: analysis of radiologic signs in 29 cases. Am. J. Roentgenol. 145: 275-281.

64. Gilchrist, A.M., M.S. Levine, R.F. Carr, et al. 1988. Barrett's esophagus: diagnosis by double-contrast esophagography. Am. J. Roentgenol. 150: 97-102.

65. Levine, M.S. 2008. Gastroesophageal reflux disease. In Textbook of Gastrointestinal Radiology, 3rd ed. R.M. Gore \& M.S. Levine, Eds.: 337-357. WB Saunders. Philadelphia.

66. Gore, R.M., J.W. Berlin, F.H. Miller, et al. 2010. Esophageal cancer. In Imaging in Oncology. J.E. Husband \& R.H. Reznek, Eds.: 127-158. Informa Healthcare. London.

67. Berkovich, G.Y., M.S. Levine \& W.T. Miller. 2000. CT findings in patients with esophagitis. Am. J. Roentgenol 175: 1431-1434.

68. Schraufnagel, D.E., J.C. Michel, T.J. Sheppard, et al. 2008. CT of the normal esophagus to define the normal air column and its extent and distribution. Am. J. Roentgenol 191: 748-752.

69. Kajander, S., A. Saraste, H. Ukkonen \& J. Knuuti. 2010 Anatomy and function: PET-CT. EuroIntervention 6(Suppl G): G87-G93.

70. Li, Y., C. Woodall, Wo J.M., et al. 2008. The use of dynamic positron emission tomography imaging for evaluating the carcinogenic progression of intestinal metaplasia to esophageal adenocarcinoma. Cancer Invest. 26: 278-285.

71. Israel, O., N. Yefremov, R. Bar-Shalom, et al. 2005. PET/CT detection of unexpected gastrointestinal foci of 18F-FDG uptake: incidence, localization patterns, and clinical significance. J. Nucl. Med. 46: 758-762.

72. Kamel, E.M., M. Thumshirn, K. Truninger, et al. Significance of incidental 18F-FDG accumulations in the gastrointestinal tract in PET/CT: correlation with endoscopic and histopathologic results. J. Nucl. Med. 45: 1804-1810.

73. Roedl, J.B., R.R. Rolen, K. King, et al. 2008 Visual PET/CT scoring for nonspecific 18F-FDG uptake in the differentiation of early malignant and benign esophageal lesions. Am. J. Roentgenol. 191: 515-521. 